\title{
High-throughput quantification of the effect of DMSO on the viability of lung and breast cancer cells using an easy-to-use spectrophotometric trypan blue-based assay
}

\author{
Sarah Musa Hammoudeh ${ }^{1}$. Arabella Musa Hammoudeh ${ }^{1} \cdot$ Rifat Hamoudi $^{1,2}$
}

Accepted: 7 February 2019 / Published online: 16 February 2019

(c) The Author(s) 2019

\begin{abstract}
One of the main aspects investigated in potential therapeutic compounds is their effect on cells viability and proliferative ability. Although various methods have been developed to investigate these aspects, these methods present with shortcomings in terms of either cost, availability, accuracy, precision, or throughput. This study describes a simple, economic, reproducible, and high-throughput assay to quantify cell death and proliferation. In this assay, adherent cells are fixed, stained with trypan blue, and measured for trypan blue internalization using a spectrophotometric absorbance plate reader. Corresponding cell counts to the absorbance measurements are extrapolated from a standard curve. This assay was used to measure the effect of dimethyl sulfoxide (DMSO) on the viability of breast and lung cancer cells. Decrease in cell count associated with the increase in DMSO percentage and exposure time. The assay's results closely correlated with the conventional trypan blue exclusion assay (Pearson correlation coefficient $(r)>0.99 ; p<0.0001$ ), but with higher precision. The assay developed in this study can be used for various applications such as optimization, cell treatment investigations, proliferation, and cytotoxicity studies.
\end{abstract}

Keywords Cell count $\cdot$ Trypan blue $\cdot$ Spectrophotometric assay $\cdot$ DMSO $\cdot$ High-throughput screening

\section{Introduction}

Cell counting is a common, important practice in cell culture used on daily basis for different applications. The increased incorporation of cell count in various techniques and assays imposed growing needs for higher levels of cell-count accuracy, precision, and throughput than those offered by the originally developed and standardized methods. Hence, various alternative approaches were developed aiming at fulfilling these requirements and overcoming original methods' shortcomings. These approaches were based on various concepts including automation, flow cytometry, impedance, tetrazolium salts, methylene blue, microscopy, fluorescence, and protein quantification.

Rifat Hamoudi

rhamoudi@sharjah.ac.ae

1 Sharjah Institute for Medical Research, College of Medicine, University of Sharjah, 27272 Sharjah, UAE

2 Department of Clinical Sciences, College of Medicine, University of Sharjah, Sharjah, UAE
Originally, the use of haemocytometers and counting chambers was the standardized cell-counting approach. However, due to their various limitations (i.e., low accuracy, low precision, low throughput, high probability of variations, and susceptibility to various human error sources), the use of these methods became limited to simple applications (Ongena et al. 2010; Tucker et al. 1994). An improved, automated modification of these methods was developed aiming at increasing the cell-count throughput. However, it failed to overcome the other shortcomings due to the similarities in sample preparation and mounting onto the special counting slides/chambers (Camacho-Fernandez et al. 2018). Furthermore, despite the various adjustment options, automated counters remain prone to fail in realizing varying cellular shapes and sizes pooled in the same sample as well as outof-focus cells in different focal planes (Camacho-Fernandez et al. 2018).

Other approaches aiming at increasing the accuracy and precision of counting were based on passing and counting each cell in the sample discretely (e.g., flow cytometry and impedance-based Coulter cell counting) (Collins et al. 2010; Coulter 1953; DeBlois and; Bean 1970). However, the use 
of these approaches requires special devices and reagents that are relatively costly and often unavailable for use. Furthermore, some of these methods focus on measuring cell number and size, but lack the ability to distinguish viable cells and cell aggregates.

Less demanding approaches with high accuracy and precision include the use of colorimetric assays (e.g., tetrazolium salt-based approaches) (Mosmann 1983; Roehm et al. 1991). However, these methods require the use of reagents and kits that are costly. Furthermore, as those approaches are based on the measurement of the colored formazan products, they are prone to artifacts affecting the $\mathrm{NAD}(\mathrm{P}) \mathrm{H}$-dependent cellular oxidoreductase enzymes' activity and formazan production (Maioli et al. 2009). These assays could as well be cell-type dependent with varying efficiencies. Colorimetric methylene blue-based counting assays were developed as simple cell-counting assays with relatively high throughput and relatively low cost (Felice et al. 2009; Oliver et al. 1989). However, the requirement of the elution step in these processes results in variations in readings and requires intensive optimization efforts (Felice et al. 2009).

In accordance with these shortcomings, we aimed to develop an easy method that would only require daily used reagents in cell culture while still providing a close efficiency to that of the other developed approaches. Applications of such an approach can vary to include proliferation studies, cytotoxicity studies, seeding count optimizations, and treatment optimizations. The capacity of this method as a cell counting and toxicity assay will be assessed through the application of DMSO to lung and breast cancer cells. DMSO is a common reagent used in various cell-culture applications including the preparation of treatments and cryopreservation. However, increased exposure to DMSO can lead to increased cell damage resulting in pseudo-effects in treatments as well as cell loss in cryopreservation.

\section{Materials and methods}

\section{Cell culture and standard curve preparation for the assay}

A549 and MDA-MB-231 cell lines (ATCC, USA) were cultured in RPMI-1640 media supplemented with 10\% FBS and $1 \%$ penicillin-streptomycin (Sigma, USA) and maintained at $37{ }^{\circ} \mathrm{C}$ and $5 \% \mathrm{CO} 2$. Cell suspension concentrations were prepared in 8 serial dilutions at a ratio of 3:4 starting with 350,000 cells $/ \mathrm{ml}$ (Table 1). Cells were then seeded in 2 sets of triplicates (one set for traditional counting and the other for the trypan blue colorimetric assay) in a 96 well plate, $100 \mu \mathrm{l}$ per well. Additional arbitrary concentrations (Table 2) were chosen to assess the efficiency of the assay in estimating cell count and were seeded with or without
Table 1 Cell concentrations seeded to constitute the standard curve

\begin{tabular}{ll}
$\begin{array}{l}\text { Con- } \\
\text { cen- } \\
\text { tra- } \\
\text { tion } \\
\text { num- } \\
\text { ber }\end{array}$ & $\begin{array}{l}\text { Standard curve serial } \\
\text { concentrations (cells/ } \\
\text { ml) }\end{array}$ \\
\hline 1 & 350,000 \\
2 & 262,500 \\
3 & 196,875 \\
4 & 147,656 \\
5 & 110,742 \\
6 & 83,056 \\
7 & 62,292 \\
8 & 46,719 \\
\hline
\end{tabular}

Following the first concentration, a ratio of $3: 4$ is used to construct the subsequent serial dilutions

Table 2 Chosen arbitrary values seeded to assess the performance of the assay

\begin{tabular}{ll}
\hline Concentration number & $\begin{array}{l}\text { Chosen arbitrary values (seeded } \\
\text { count (cells/ml) } \pm \text { DMSO treat- } \\
\text { ment) }\end{array}$ \\
\hline 1 & $200,000-$ DMSO \\
2 & $200,000+$ DMSO \\
3 & $150,000-$ DMSO \\
4 & $150,000+$ DMSO \\
5 & $100,000-$ DMSO \\
6 & $100,000+$ DMSO \\
7 & $50,000-$ DMSO \\
8 & $50,000+$ DMSO \\
\hline
\end{tabular}

Each concentration is seeded with $(+)$ or without $(-)$ DMSO treatment to investigate the potential use of the assay for cytotoxicity studies

5\% dimethyl sulfoxide (DMSO; Sigma, USA), similarly in triplicates. The 96-well plate was left for 10-15 $\mathrm{min}$ in the biological cabinet before transferring to the incubator to avoid aggregation of cells in the middle of the well. A549 cells were incubated for $6 \mathrm{~h}$, whereas MDA-MB-231 cells were incubated for $20 \mathrm{~h}$. Cells were monitored throughout for adherence and normal morphology adaptation and the experiment was stopped (fixation/trypsinization) once these conditions were fulfilled.

\section{Traditional trypan blue cell counting}

Cells were harvested with $0.05 \%$ Trypsin-EDTA (Sigma, USA) and enzymatic activity was neutralized with FBScontaining RPMI-1640 Media. Cell densities were measured using a haemocytometer after the addition of trypan blue. 
Densities were then adjusted for the final count per well by multiplying by the suspension volume in each well.

\section{Trypan blue staining}

The cells were fixed with 4\% Paraformaldehyde (PFA) (Sigma, USA) (prepared in PBS) for $20 \mathrm{~min}$ at room temperature followed by 2 washed with PBS. The cells were then stained with $0.1,0.25$, or $0.4 \%$ Trypan Blue (Sigma, USA) diluted in PBS for 10, 30, or 60 min at room temperature. The cells were then washed twice with PBS to completely remove any remaining trypan blue residues and avoid an artifact signal. The absorbance was then measured using BioTek's plate reader ELx808 at absorbance of $450 \mathrm{~nm}$, $490 \mathrm{~nm}$, and $630 \mathrm{~nm}$.

\section{Microscopy}

Some of the images were acquired with Olympus IX53 inverted microscope equipped with Olympus DP75 camera (resolution of $5760 \times 3600$ pixels and pixel size of $5.86 \times 5.86 \mu \mathrm{m}$ ) with Cell Sens Entry software (version 1.17). Images were acquired with $10 \mathrm{X}$ (numerical aperture 0.50 ) objective lenses at $1600 \times 1200$ pixels and $72.01 /$ in resolution in both $\mathrm{X}$ and $\mathrm{Y}$ axis. The rest of the images were acquired using Olympus IX73 inverted microscope with Olympus DP22 camera (resolution of $1920 \times 1440$ pixels and pixel size of $3.69 \times 3.69 \mu \mathrm{m})$ and DP camera acquisition software. Images were acquired with $4 \mathrm{X}$ (numerical aperture 0.13 ) or at $1920 \times 1440$ pixels resolution.

\section{Statistical analysis}

$T$ test and one-way ANOVA tests were conducted to statistically analyze the cell-count data and identify differences between the results of the different counting methods. The significance was taken to be $p<0.05$. Excel was used to conduct the goodness-of-fit test $\left(R^{2}\right)$ as a part of the standard curve linear regression analysis. Otherwise, all statistical analyses (including Pearson's correlation) were carried out using GraphPad Prism.

\section{Results}

\section{Optimization of the trypan blue assay parameters}

The use of this assay requires the optimization of multiple parameters: (1) standard curve range and ratio of serial dilutions; (2) standard curve plating duration; and (3) duration of fixing and staining.

To optimize the standard curve range, multiple concentrations of cells approaching the two extremes (full confluency and very low confluency) were plated in a 96-well plate. Accordingly, the rough borderlines of the standard curve range were determined, $\sim 35,000$ cells/well at full confluency and $\sim 5000$ cells/well at low confluency, for both cell lines. This range was then used to identify the dynamic range of the assay by testing its accuracy at the two previously identified borderlines. Subsequently, a serial dilution ratio (3:4 dilution starting from 35,000 cells/well) was chosen to create a standard curve spanning the chosen range (Table 1). The cells were plated according to the dilutions and monitored regularly for signs of adherence and adaptation of natural morphology in culture. Once the cells fulfilled these conditions (after $6 \mathrm{~h}$ for A549 cells and $20 \mathrm{~h}$ for MDA cells), the cells are directly fixed for staining to avoid increase in cell count.

Beyond fixing the cells, the application of PFA sufficiently permeabilizes the cell membrane to allow for the entrapment of trypan blue intracellularly and the full coverage of the cell body (Fig. 1). This method of staining results in dye density signals proportional to the volume

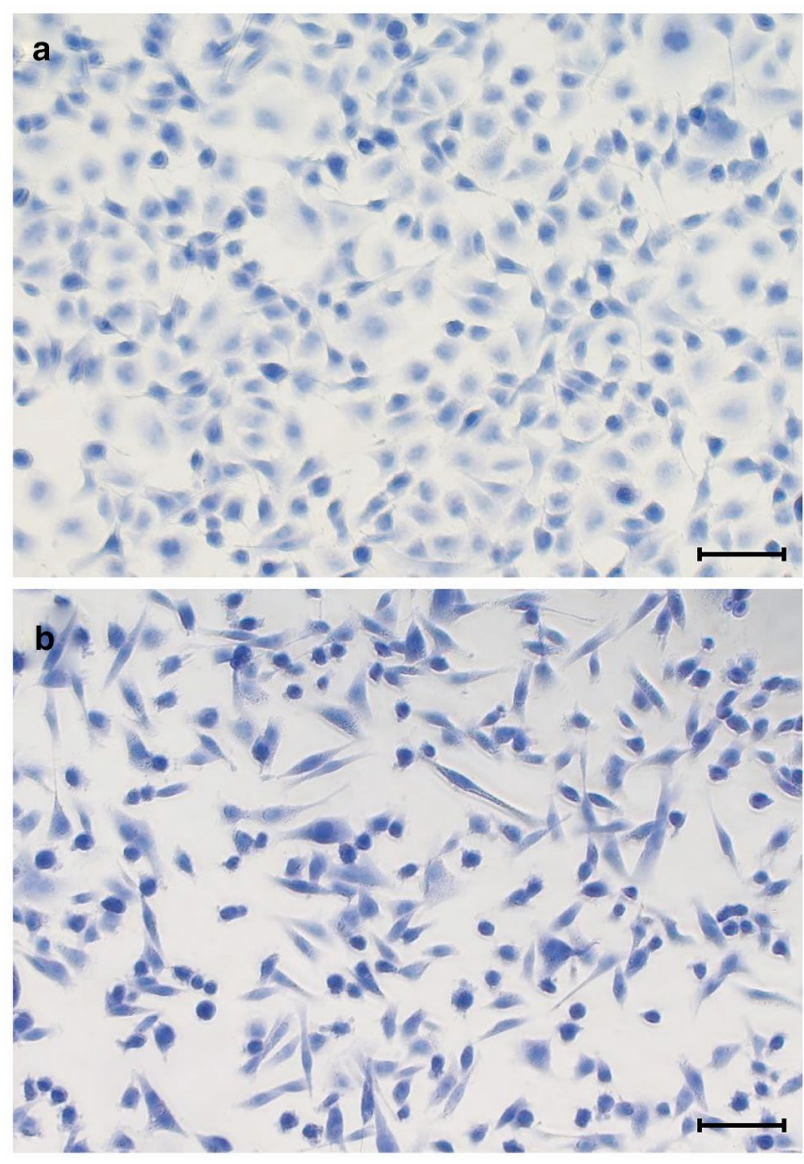

Fig. 1 Dye absorption by a A549 and b MDA-MB-231 cells after fixation and staining with trypan blue. Scale bars representing $100 \mu \mathrm{m}$. Images acquired with 10X magnification through Olympus IX53 inverted microscope 
and thickness of the cell; rounded cells are less spread but thicker resulting in a darker shade of blue and the opposite for cells that adapted to the normal morphology. This balance between color density and surface area coverage compensates for differences in cell morphologies and allows for a better cell-count estimation.

Staining duration was optimized over $0.5,1,2,5 \mathrm{~h}$, and overnight. As shown in Fig. 2, although the trypan blue signal could be visualized even at shorter staining duration, the strength of the signal directly correlated with the staining duration. Therefore, to achieve the best visualization of the cells, overnight staining was applied to the subsequent experiments. Inefficiencies in the trypan blue internalization into the cells might require optimization of the fixation method and staining duration. Otherwise, one might consider the application of a highly diluted detergent (e.g.,

a

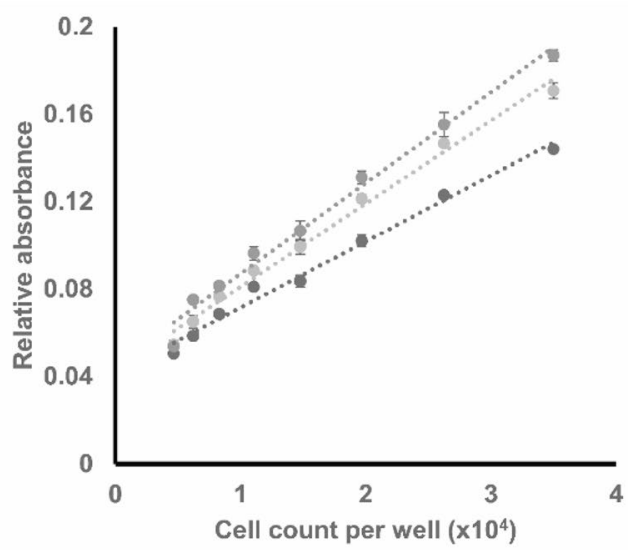

$\bullet 0.1 \%$ trypan blue $\quad 0.25 \%$ trypan blue $0.4 \%$ trypan blue

b

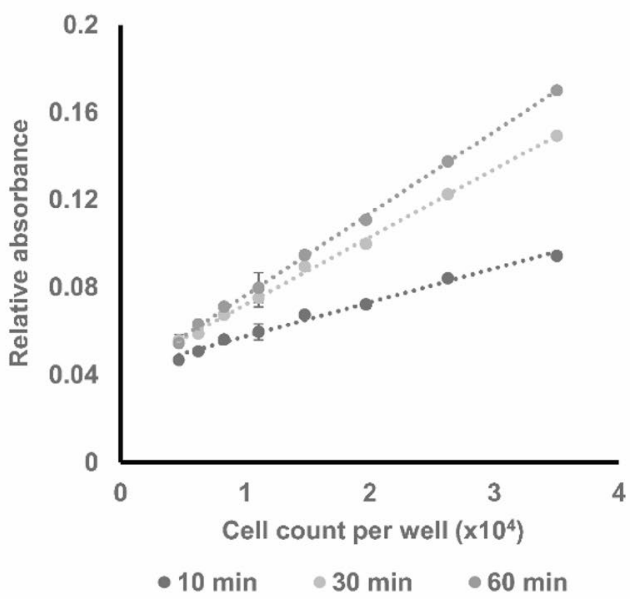

Fig. 2 Optimization of trypan blue staining parameters including a trypan blue concentration $(0.1,0.25$, and $0.4 \%$ trypan blue), b staining duration $(10,30$, and $60 \mathrm{~min})$, $\mathbf{c}$ pre-permeabilization with $0.5 \%$
Triton X-100) to lightly permeabilize cell membrane and increase dye internalization.

To achieve the optimum staining procedure, several parameters were optimized (Fig. 2). Initially, the staining resulting from ascending concentrations of trypan blue $(0.1,0.25$, and $0.4 \%)$ was investigated showing a positive concordance between the concentration of trypan blue and dye absorption (Fig. 2a). With the use of the highest concentration, $0.4 \%$ of trypan blue, the staining duration was optimized over 10, 30, and $60 \mathrm{~min}$ (Fig. 2b). Positive correlation was similarly observed between the staining duration and dye absorption. The additional permeabilization of the cells with $0.5 \%$ Triton-X100 prior to the staining was found to further increase trypan blue uptake (Fig. 2c). Therefore, permeabilization with Triton X-100 can be used to reduce the staining duration; $30 \mathrm{~min}$ dye uptake by Triton X-100 permeabilized cells closely approaches that of

C

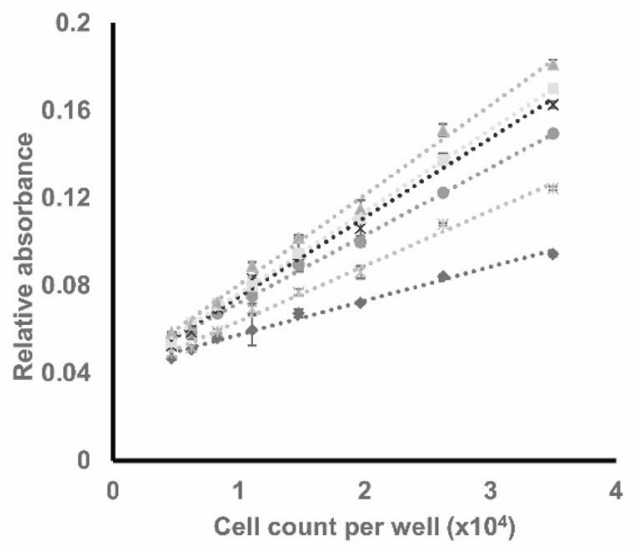

- 10 minutes (-) Triton X-100 $* 10$ minutes (+) Triton X-100

- 30 minutes (-) Triton X-100 $\times 30$ minutes (+) Triton X-100

$=60$ minutes $(-)$ Triton X-100 $\triangle 60$ minutes (+) Triton X-100

d

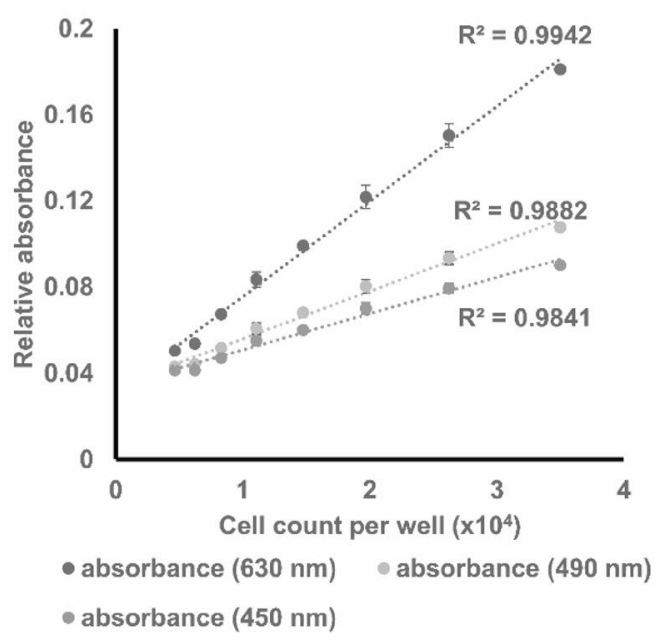

Triton X-100, and d absorbance acquisition wavelength. Data points presented as mean \pm SEM 
non-permeabilized cells stained for $1 \mathrm{~h}$. Taken together, the optimum staining conditions were found to be staining with $0.4 \%$ trypan blue at room temperature for $30 \mathrm{~min}$ if preceded by 20 min of Triton X-100 permeabilization or $1 \mathrm{~h}$ without permeabilization.

Furthermore, absorbance measurement at the three different wavelengths $(450,490$, and $630 \mathrm{~nm})$ was found to provide sufficient signal to estimate cell count, indicating the flexibility of the assay (Fig. 2d). However, as the absorbance measurement at wavelength of $630 \mathrm{~nm}$ provided the strongest signal, and the highest correlation and accuracy, it was used in this paper for all subsequent measurements.
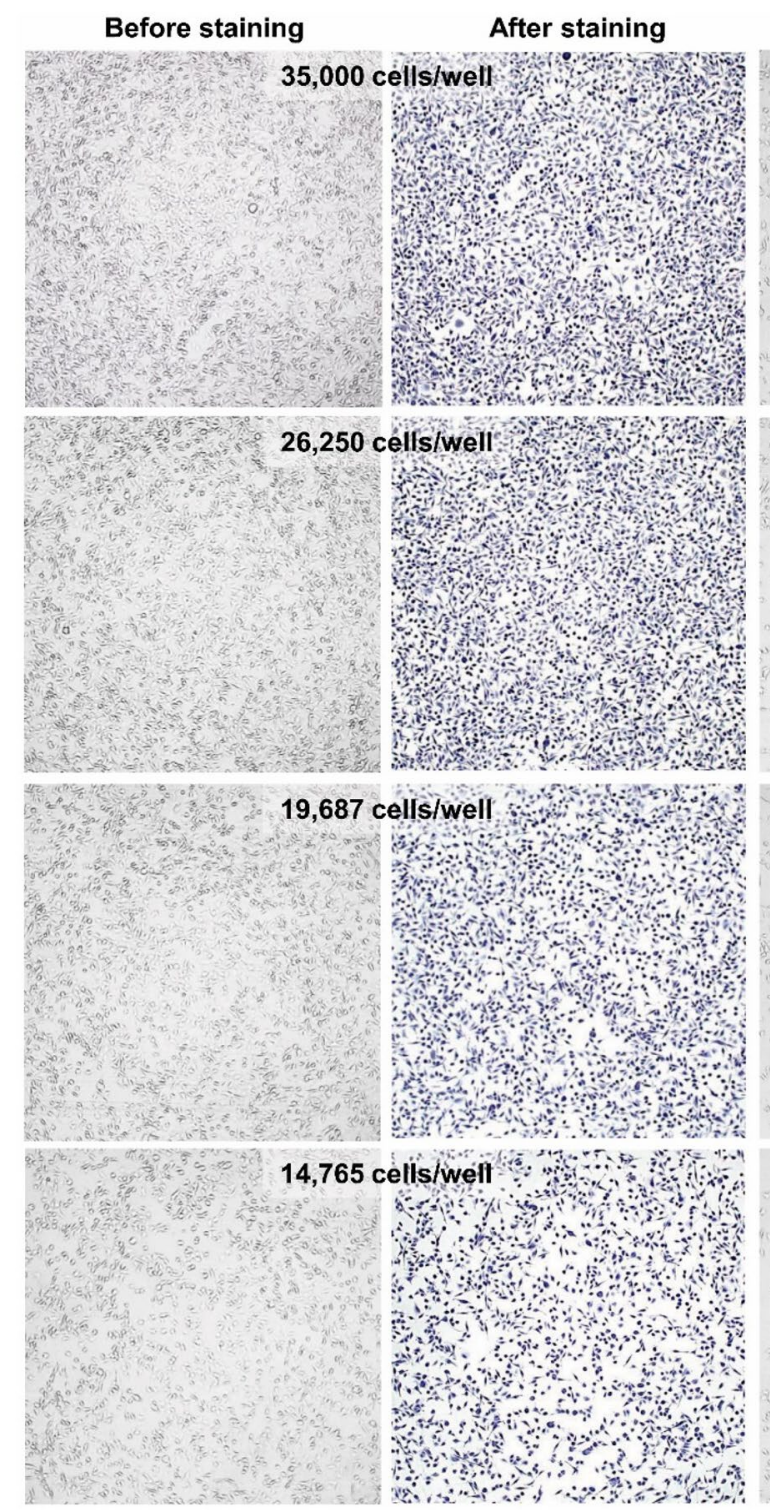

\section{Standard curve accuracy verification}

The gradual reduction in cell density along the standard curve serial dilutions and the corresponding decrease in trypan blue staining were visually verified using phase contrast microscopy (Fig. 3). The trypan blue absorbance signal was then quantified using BioTek's plate reader Elx808. As seen in the absorbance measurements of the standard curves for both cell lines (Fig. 4a, b), a significant correlation [correlation results for the A549 cell line: Pearson's correlation: 0.9971; $p<0.0001$; goodness of fit $\left(R^{2}\right)$ : 0.9942; correlation results for the MDA-MB-231 cell line: Pearson's correlation: 0.9919, $p<0.0001$; goodness of fit

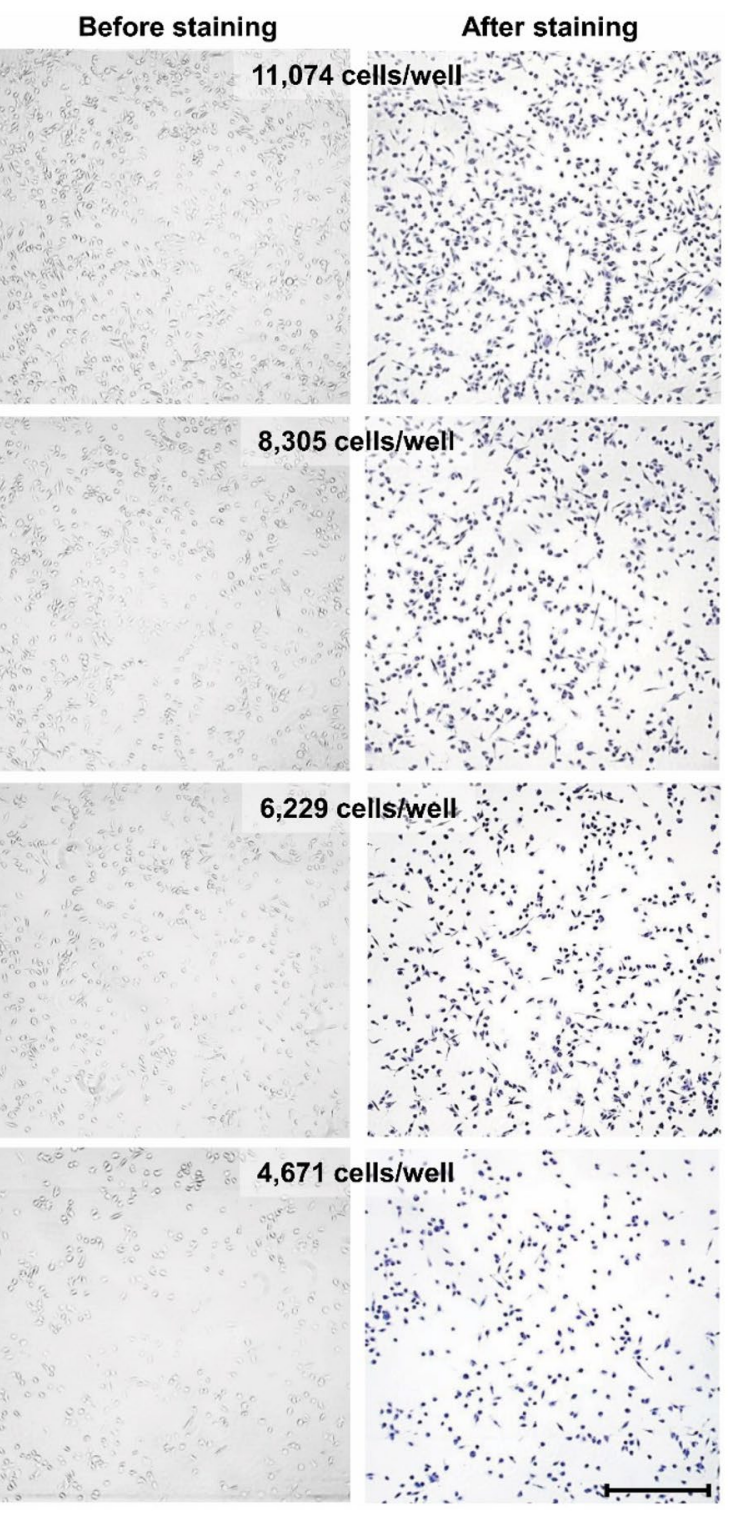

Fig. 3 Standard curve serial densities of A549 cells before and after fixation and staining with trypan blue. Scale bars representing $200 \mu \mathrm{m}$. Images were taken at a 4X magnification using Olympus IX73 inverted microscope 


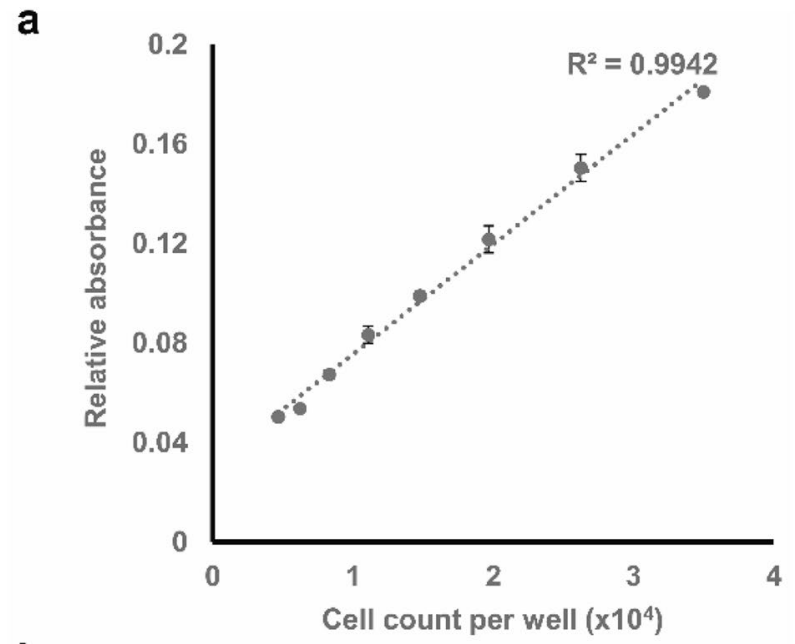

b

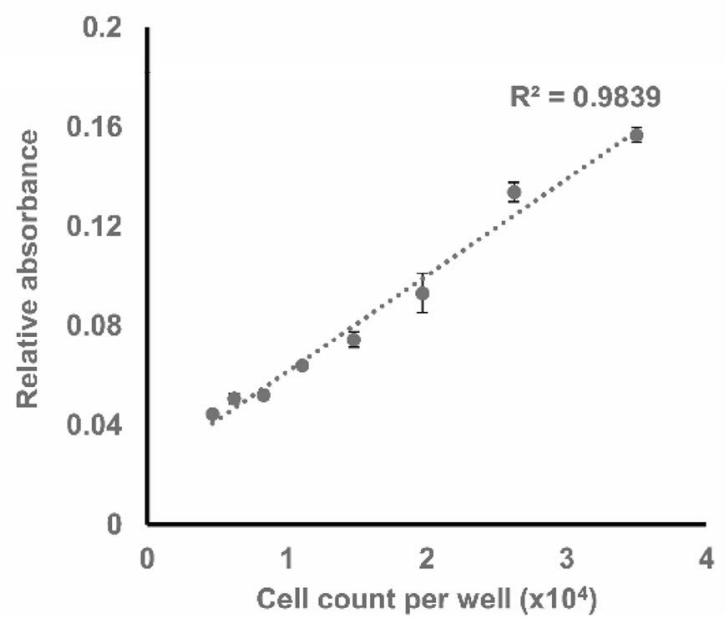

Fig. 4 Absorbance standard curve and goodness-of-fit test $\left(R^{2}\right)$ as a measure of correlation for a A549 and b MDA-MB-231 cells. Data points presented as mean \pm SEM

$\left.\left(R^{2}\right): 0.9839\right]$ is achieved with low variations amongst the triplicate measures at each point.

\section{Comparison between cell-count measurements using the conventional haemocytometer and the new trypan blue assay}

Standard curve cell-count measurements were similarly done using the traditional haemocytometer counting method. Several precautions were taken to increase the accuracy and precision of these counts including: (1) ensuring proper detachment of cells and homogenous suspension; (2) conducting triplicate measurements at each point; (3) counting cells in the four outer edges squares of the haemocytometer grid; and (4) including cells only on the top and right edges of each of these squares (Fig. 5a). Furthermore, images were taken of the haemocytometer grid instead of instantaneous counting to avoid cell damage due to prolonged cell incubation at room temperature.

These cell-count measurements were then compared to those of the new trypan blue colorimetric assay. As can be seen in the graphs (Fig. 5b, c), the measurement from both methods was relatively close and fluctuates narrowly around the initially seeded counts. However, in comparison with the haemocytometer's measurements, the counts from the new assay were found to be more representative of the initially seeded cell counts and more precise in terms of triplicate measurements. This indicates the comparatively higher accuracy and precision of the new trypan blue colorimetric assay.

\section{Efficiency of the assay in estimating arbitrary samples with unknown counts}

Arbitrary values were chosen to assess the power of the assay and its ability to estimate unknown cell counts. As seen in the graphs of the two cell lines (Fig. 6), the results from the two assays fluctuate closely around the initially seeded counts. However, at multiple data points, the trypan blue assay proved to yield closer cell counts to those initially seeded. Furthermore, the ranges of inter-triplicate variations are significantly smaller in the trypan blue spectrophotometric assay's measurements.

\section{Cytotoxicity assay}

The arbitrarily chosen values above were treated with 5\% DMSO to assess the ability of the trypan blue spectrophotometric assay in measuring treatment-induced cytotoxicity. The assay measurements of the DMSO treated cells (Fig. 7) show a clear reduction in cell count, conforming to the results of the standard haemocytometer counting.

Furthermore, the resolution power of the assay was assessed by measuring the cytotoxic effect of 3 consecutive concentrations of DMSO, 1, 2, 3, 4, 5, 7.5, and 10\% (Fig. 8a). Starting at an initial count of $\sim 40,000$ cells per well, a gradual decrease is seen in cell count in correspondence with the increase in the applied DMSO concentration. Hence, this demonstrates the ability of the assay to detect and differentiate between relatively close cell counts. Moreover, the cell-count measurements obtained with the new trypan blue assay positively correlated with those from traditional haemocytometer counting (Pearson's correlation coefficient: $0.9771 ; p<0.0001 ; R^{2}: 0.9546$ ), verifying the validity of the new assay's measurements. 
a

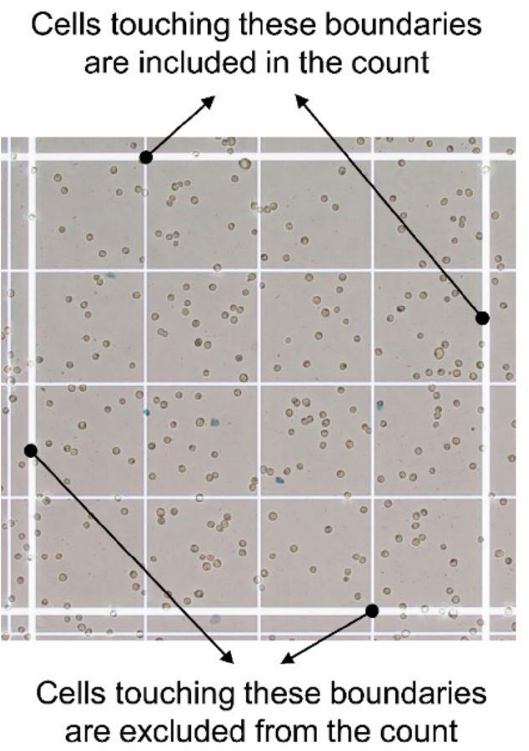

b

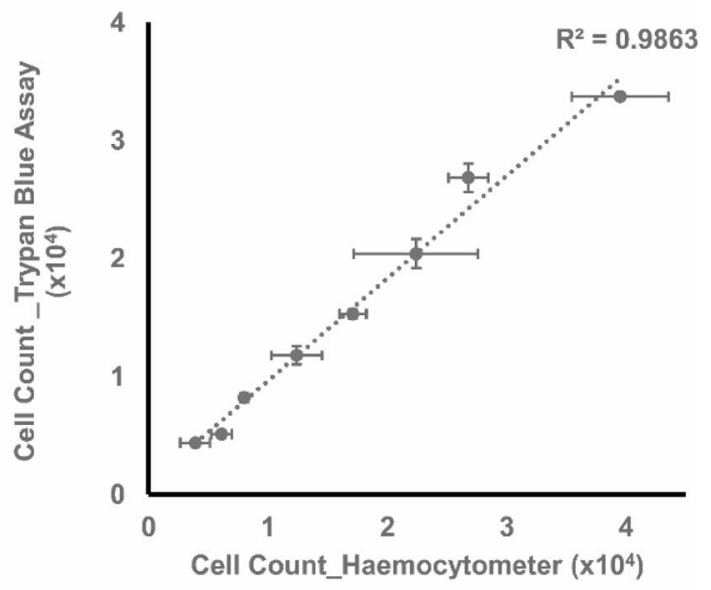

C

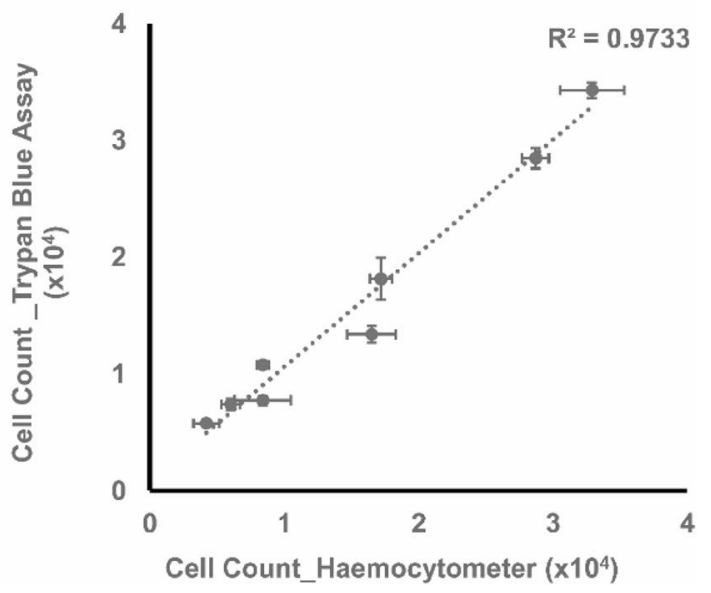

obtained using haemocytometer and the new trypan blue assay. Data points presented as mean \pm SEM (horizontal error bars representing SEM of haemocytometer count while the vertical ones representing that of the new trypan blue assay)

\section{Discussion}

Although various cell-counting approaches were developed to fulfil application-specific needs, these approaches are either low throughput (e.g., haemocytometer) or relatively cost-demanding (e.g., flow cytometry). In this paper, we present a simple, cost-efficient, time-saving, high throughput, and reasonably precise and accurate assay. The concept behind this assay is to measure cell density by measuring the internalization of trypan blue by fixed, adherent cells. This measurement is then used to infer the cell number in the well by plotting to a standard curve.

As shown in the results' section, the proposed trypan blue assay provided close estimation of the arbitrarily selected cell counts. Moreover, it efficiently captured the cytotoxic effect of DMSO treatment on cells which positively correlated with the increase in the applied DMSO percentage. The negative effect of DMSO on cell viability was found as well to correlate with the exposure duration. 10\% DMSO 
a

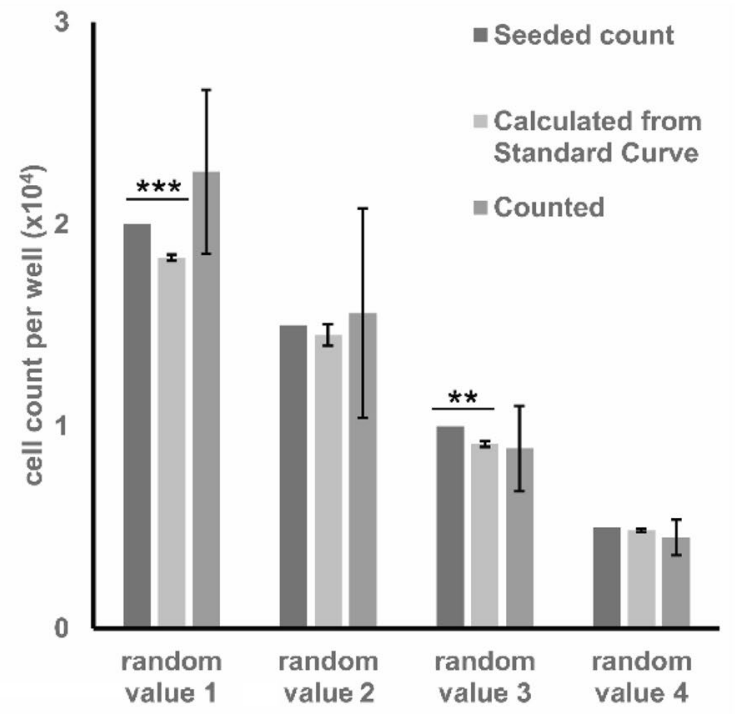

b

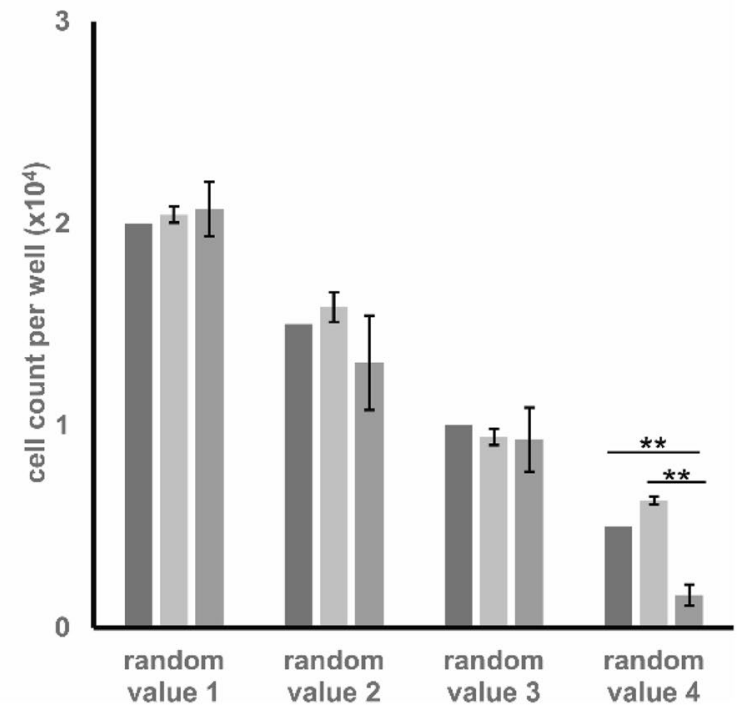

Fig. 6 Cell-count measurements of arbitrary seeded counts of a A549 and b MDA-MB-231 cells using traditional haemocytometer counting (counted) and the new trypan blue assay (calculated from standard curve) in comparison with originally seeded counts. Data points presented as mean $\pm \mathrm{SEM}$; **represents $p \leq 0.01$, and $* * *$ represents $p \leq 0.001$

is a commonly used concentration in preparation of cells for cryopreservation. However, prolonged exposure to this percentage is shown above to induce cell damage proportional to the exposure duration. These results reflect the previous findings on the various mechanisms stimulated by DMSO to induce cell damage in correspondence to its concentration. For instance, DMSO application at concentrations $>10 \%$ results in the perforation of the plasma membrane and eventually apoptosis (De Ménorval MA 2010; Notman 2006). On the other hand, lower concentrations of DMSO were found to result in the nuclear translocation of

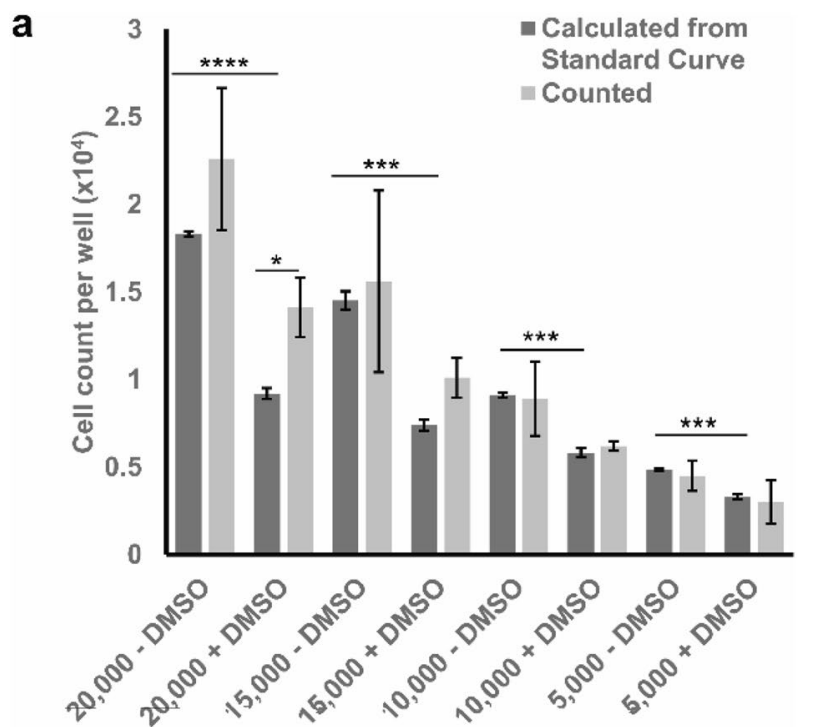

b

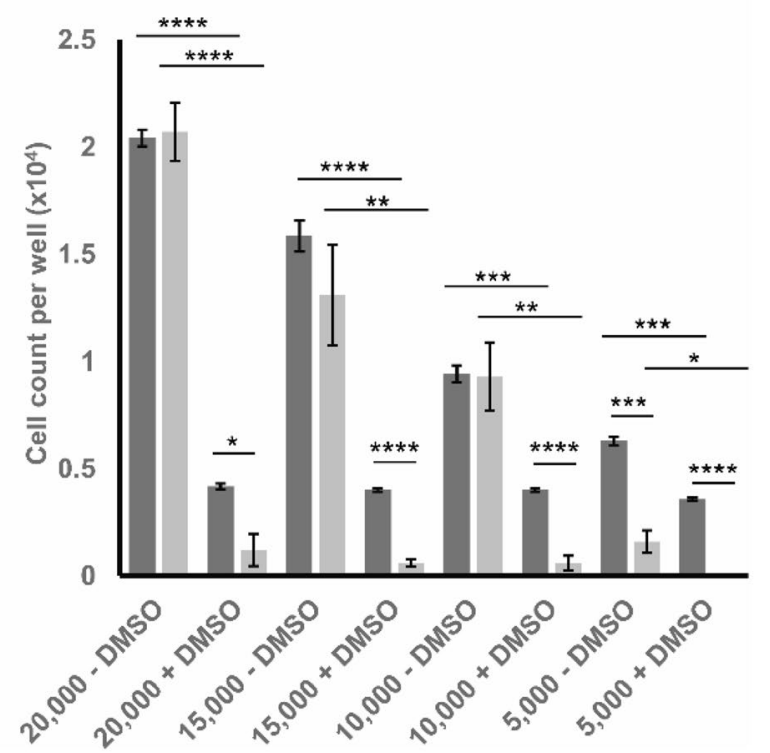

Fig. 7 Cell-count measurements of the chosen arbitrary cell counts after treatment with 5\% DMSO calculations for a A549 cell line and b MDA-MB-231 cell line using traditional haemocytometer counting (counted) and the new trypan blue assay (calculated from standard curve). Data points presented as mean \pm SEM; *represents $p \leq 0.05$, $* *$ represents $p \leq 0.01$, *** represents $p \leq 0.001$, and $* * * *$ represents $p \leq 0.0001$

the apoptosis-induced factor (AIF) from the mitochondria, activation of poly-(ADP-ribose)-polymerase (PARP), and stimulation of caspase-3-independent apoptosis pathways (Galvao 2014).

In comparison with the haemocytometer standardized counting, the trypan blue colorimetric assay counting results are relatively higher in accuracy, precision, and resolution. Furthermore, the assay only requires manual counting of a single value (the highest value in the standard curve) which would be used as the reference count for all subsequent 
a

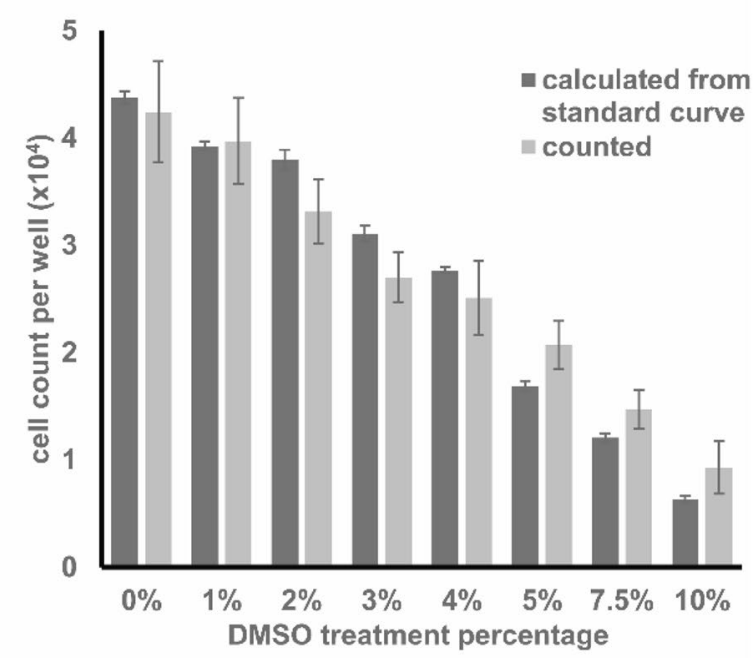

b

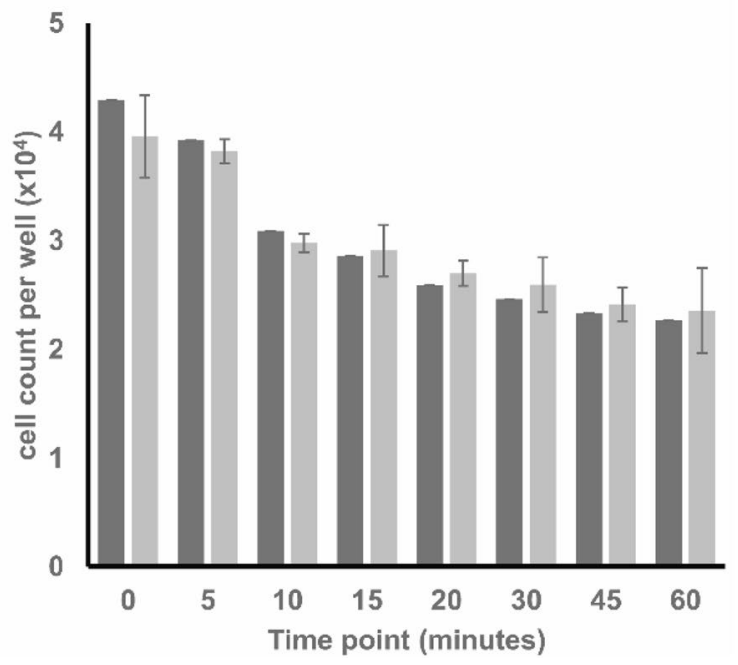

Fig. 8 Cell-count measurements of A549 cells treated with a 0, 1, $2,3,4,5,7.5$, and $10 \%$ of DMSO for $24 \mathrm{~h}$ and $\mathbf{b} 10 \%$ DMSO for increasing time periods $(0-60 \mathrm{~min})$ measured with the traditional hemocytometer counting (counted) and the new trypan blue assay (calculated from standard curve). Data points presented as mean \pm SEM

calculations. Hence, the assay has a significantly increased throughput and time efficiency. Standard counting with haemocytometer, as well as many of the other approaches, requires the trypsinization and detachment of cells. This process increases the risk of cell loss and affects cell count. In this approach, we eliminated this risk by directly staining and measuring the absorbance on the same cell-culture surface of the 96-well plate.

Moreover, the use of this assay requires reagents used daily in cell-culture practice (i.e., trypan blue and paraformaldehyde) and a simple spectrophotometric plate reader, usually at disposal to most. Therefore, the assay is developed to be user-friendly, economic, and suitable for frequent, simple counting procedures (i.e., optimization experiments, proliferation, and cytotoxicity assessment). To further increase the cost efficiency of the assay, the diluted trypan blue dye can be recycled from the previous experiments. Another advantage of this approach is that in case of weak trypan blue signal, the cells can be simply re-incubated with the trypan blue dye for a longer time period. Furthermore, the experiment plate stained with the trypan blue can be saved for back-referencing by storing it at $4{ }^{\circ} \mathrm{C}$ in PBS with the option of re-staining in case dye is eluted.

Due to the cell-volume dependence of the assay, the measurements done through this assay are relatively reproducible. This results in various advantages; for example, the optimized standard curve could be reused for the calculations for subsequent assays on the condition that the fixation and staining processes are identical. This is especially the case with stable cell lines with uniform morphology, adherence, and growth rates. Furthermore, cell populations with irregular shapes would be accounted for in this assay. Shape irregularities will result in changes in cell thickness which will be directly accounted for by changes the trypan blue signal (positive correlation between thickness and signal). Moreover, it might be possible to use the assay for counting cell populations heterogeneous in volume and shape as these differences will be considered in the standard curve and reflect on the count of the target samples; this require on proper homogenous distribution of these cells throughout the suspension. Third, overlapping or clustering of seeded cells would result in a correlative increase in dye intensity. Thus, the overlapped cells would be as well accounted for in the final count of cells per well.

Successfully conducting this assay requires several precautions and requirements: (1) ensuring homogenous distribution of cells on the well surface by allowing the cells to settle and adhere to the plate before transferring it to the incubator; (2) pipetting gently to avoid cell loss due to mechanical pressure; and (3) reducing the incubation time of the cells in suspension before plating to enhance cell adhesion and ensure proper morphology adaptation.

Although this approach has the shortcoming of being a fixed-cell assay, its value lies in its potential use as a proliferation/cytotoxicity assay similarly to, but at a lower cost than currently existing colorimetric assays (e.g., tetrazolium salt-based approaches). The use of this approach can be ideal for optimization procedures requiring multiple repetitions and assessing multiple parameters with wide ranges. In such cases, the use of the relatively expensive approaches would result in a high economic burden. While economic options, such as the haemocytometer, would be impractical due to its low throughput and potential time consumption. Accordingly, this assay can provide measurements relatively better than those of 
the haemocytometer and at a faster rate and economic cost. Moreover, this assay could potentially be modified and expanded to other cell viability exclusion dyes with similar properties to those of trypan blue according to their availability in the lab, further increasing the feasibility of the assay.

Acknowledgements R.H. is funded by Al Jalila Foundation (AJF201741), University of Sharjah and Boehringer Ingelheim (120102). We thank Dangoor Education for supporting this work.

OpenAccess This article is distributed under the terms of the Creative Commons Attribution 4.0 International License (http://creativeco mmons.org/licenses/by/4.0/), which permits unrestricted use, distribution, and reproduction in any medium, provided you give appropriate credit to the original author(s) and the source, provide a link to the Creative Commons license, and indicate if changes were made.

\section{References}

Camacho-Fernandez C, Hervas D, Rivas-Sendra A, Marin MP, SeguiSimarro JM (2018) Comparison of six different methods to calculate cell densities. Plant Methods 14:018-0297

Collins CE, Young NA, Flaherty DK, Airey DC, Kaas JH (2010) A rapid and reliable method of counting neurons and other cells in brain tissue: a comparison of flow cytometry and manual counting methods. Front Neuroanat 4:55

Coulter WH (1953) Means for counting particles suspended in a fluid. US Patent, 1953

De Ménorval MAML, Fernández ML, Reigada R (2010) Effects of dimethyl sulfoxide in cholesterol-containing lipid membranes: a comparative study of experiments in silico and with cells. PLoS One 7:e41733
DeBlois R W, Bean P C (1970) Counting and sizing of submicron particles by the resistive pulse technique. Rev Sci Instrum 41(7):909_ 916. https://doi.org/10.1063/1.1684724

Felice DL, Sun J, Liu RH (2009) A modified methylene blue assay for accurate cell counting. J Funct Foods 1:109-118. https://doi. org/10.1016/j.jff.2008.09.014

Galvao JDB, Tilley M, Normando E, Duchen MR, Cordeiro MF (2014) Unexpected low-dose toxicity of the universal solvent DMSO. FASEB J 28:1317-1330

Maioli E, Torricelli C, Fortino V, Carlucci F, Tommassini V, Pacini A (2009) Critical appraisal of the MTT assay in the presence of rottlerin and uncouplers. Biol Proced Online 11:227-240

Mosmann T (1983) Rapid colorimetric assay for cellular growth and survival: application to proliferation and cytotoxicity assays. J Immunol Methods 65:55-63

Notman RNM, O'Malley B, Anwar J (2006) Molecular basis for dimethylsulfoxide (DMSO) action on lipid membranes. J Am Chem Soc 128:13982-13983

Oliver MH, Harrison NK, Bishop JE, Cole PJ, Laurent GJ (1989) A rapid and convenient assay for counting cells cultured in microwell plates: application for assessment of growth factors. J Cell Sci 92:513-518

Ongena K, Das C, Smith JL, Gil S, Johnston G (2010) Determining cell number during cell culture using the Scepter cell counter. J Vis $\operatorname{Exp} 26: 6$

Roehm NW, Rodgers GH, Hatfield SM, Glasebrook AL (1991) An improved colorimetric assay for cell proliferation and viability utilizing the tetrazolium salt XTT. J Immunol Methods 142:257-265

Tucker KG, Chalder S, al-Rubeai M, Thomas CR (1994) Measurement of hybridoma cell number, viability, and morphology using fully automated image analysis. Enzyme Microb Technol 16:29-35

Publisher's Note Springer Nature remains neutral with regard to jurisdictional claims in published maps and institutional affiliations. 